\title{
Towards a dephasing diode: asymmetric and geometric dephasing
}

\author{
Robert S. Whitney, ${ }^{1}$ Alexander Shnirman, ${ }^{2,3}$ and Yuval Gefen ${ }^{4}$ \\ ${ }^{1}$ Institut Laue-Langevin, 6 rue Jules Horowitz, B.P. 156, 38042 Grenoble, France. \\ ${ }^{2}$ Institut für Theoretische Festkörperphysik and DFG-Center for Functional Nanostructures (CFN), \\ Universität Karlsruhe, D-76128 Karlsruhe, Germany. \\ 3 Institut für Theoretische Physik, Universität Innsbruck, Technikerstr. 25, 6020 Innsbruck, Austria. \\ ${ }^{4}$ Department of Condensed Matter Physics, The Weizmann Institute of Science, Rehovot 76100, Israel.
}

(Dated: March 25, 2008)

\begin{abstract}
We study the effect of a noisy environment on spin and charge transport in ballistic quantum wires with spin-orbit coupling (Rashba coupling). We find that the wire then acts as a dephasing diode, inducing very different dephasing of the spins of right and left movers. We also show how Berry phase (geometric phase) in a curved wire can induce such asymmetric dephasing, in addition to purely geometric dephasing. We propose ways to measure these effects through spin detectors, spin-echo techniques, and Aharanov-Bohm interferometry.
\end{abstract}

PACS numbers: 03.65.Yz, 85.75.-d, 73.63.Nm, 73.23.Ad.

A very promising idea for future (quantum or classical) information processing is "spintronics" 1, 2, 2, 3, 4], where electrons' spins (not their charges) are used to encode information. However spins do not obey the same conservation laws as charges; charges do not change sign but spins can flip. Current conservation enforces symmetries on charge transport (Onsager relations). For example two-terminal devices always have the same left-toright and right-to-left conductance in the linear-response regime of negligible interaction/charging effects (diodes do not exist without interactions). By contrast, asymmetries between left-to-right and right-to-left spin-transport can occur in the linear-response regime for two-terminal devices, if there is spin-orbit coupling (Rashba or Dresselhaus). Coherence is a crucial aspect of quantum transport, so here we investigate analogous asymmetries in the dephasing (decay of coherence) of spins. We then study how Berry (geometric) phases - present in curved wires [5] - modify such asymmetric dephasing [6].

The coherence of a superposition of two spin-states at the Fermi-surface is quantified in terms of the purity, $P=\operatorname{tr}\left[\hat{\rho}^{2}\right]$, where $\hat{\rho}$ is a $2 \times 2$ density matrix. A pure superposition has maximal purity, $P=1$, while an equal classical mixture has minimal purity, $P=1 / 2$. We use the term "dephasing diode" for a two-terminal device in which spin-superpositions of left movers experience very different dephasing from right movers. For an ideal dephasing diode, an electron injected into the device from the left lead (a right mover) in an equal coherent superposition of spin-states $(P=1)$ would emerge completely dephased $(P=1 / 2)$, while an electron injected into the device from the right (a left mover) in any superposition with $P=1$ would emerge at the left without being dephased at all (still having $P=1$ ). This dephasing could be observed by measuring either certain spin components of the current, or by a conventional current measurement in an Aharonov-Bohm (AB) interferometer. In this letter, we provide illustrations of dephasing diodes with straight and curved ballistic wires. For the latter, the Berry phase gives a geometry-induced contribution to dephasing, whose sign depends on the curvature's sign.

Noise causes dephasing, and real devices have many sources of noise (thermal or quantum), including electron-electron and electron-phonon interactions. However, a clear experimental observation of asymmetric dephasing requires control of the noise-power (seeing the asymmetry change with the noise-power). Thus we propose taking a wire with low intrinsic noise, modelled by ballistic non-interacting electrons, and applying manmade noise to the magnetic fields and gates. The response time of gates/magnets is typically longer than the time-of-flight of electrons from source to detector along a ballistic wire 7]. Hence we study the effect of extremely slow (man-made or intrinsic) noise on noninteracting electrons with spin-orbit coupling. For simplicity, here we consider only Rashba coupling, $\mathbf{B}_{\mathrm{R}}=$ $\hbar\left(\hat{p}_{x} \mathbf{e}_{y}-\hat{p}_{y} \mathbf{e}_{x}\right) /\left(m l_{\mathrm{R}}\right)$, where $l_{\mathrm{R}}$ is the spin-precession length [8], and magnetic fields are in units of energy. Biasing a back-gate gives control over $l_{\mathrm{R}}$ by modifying the potential gradient along the $z$-axis [9]. Noise can be applied to either the applied magnetic-field or the Rashba spin-orbit coupling (via a noisy voltage on the backgate). By measuring any three orthogonal spin polarizations $\left\langle\sigma_{i}\right\rangle, i \in\{1,2,3\}$, one gets the purity $P=\frac{1}{2}\left(1+\left\langle\hat{\sigma}_{1}\right\rangle^{2}+\left\langle\hat{\sigma}_{2}\right\rangle^{2}+\left\langle\hat{\sigma}_{3}\right\rangle^{2}\right)$ [10]. Our qualitative predictions are summarized in Table \ Dresselhaus coupling yields similar results, but it is hard to control experimentally (i.e. not affected by the back-gate) so we do not consider it further here [11].

Asymmetric dephasing in a straight wire (see Fig. 1. $)$. We neglect the contribution of the motion across the wire ( $y$-direction) [12], so the Hamiltonian for an electron in the lowest mode of the ballistic wire is

$$
\hat{\mathcal{H}}=(2 m)^{-1} \hat{p}_{x}^{2}+E_{0}-\hbar\left(m l_{\mathrm{R}}\right)^{-1} \hat{p}_{x} \hat{\sigma}_{y}-\frac{1}{2} \mathbf{B} \cdot \hat{\sigma},
$$

where $E_{0}$ is the transverse mode's energy. Hence the 
(a) straight wire with in-plane applied field

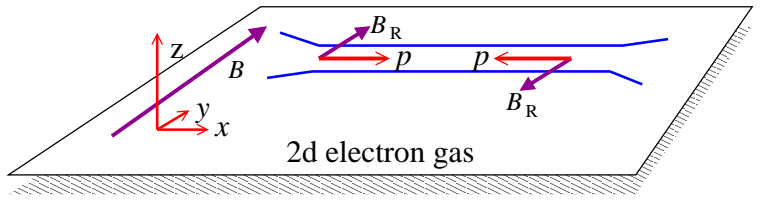

(b) curved wire with perpendicular applied field

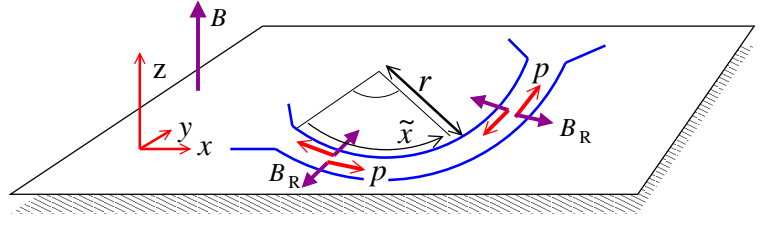

(c) curved wires with the same geometric phase/dephasing

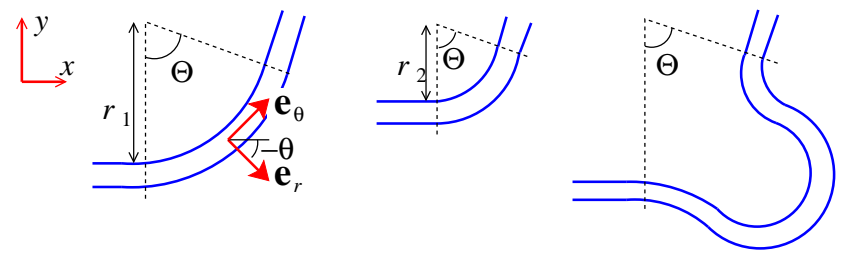

(d) spin-echo set-up with a curved wire then a straight wire

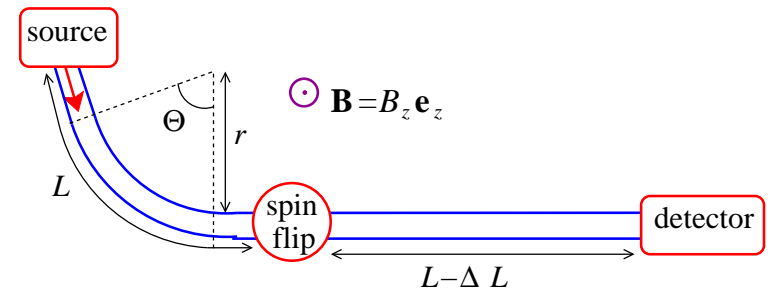

(e) Aharonov-Bohm set-up

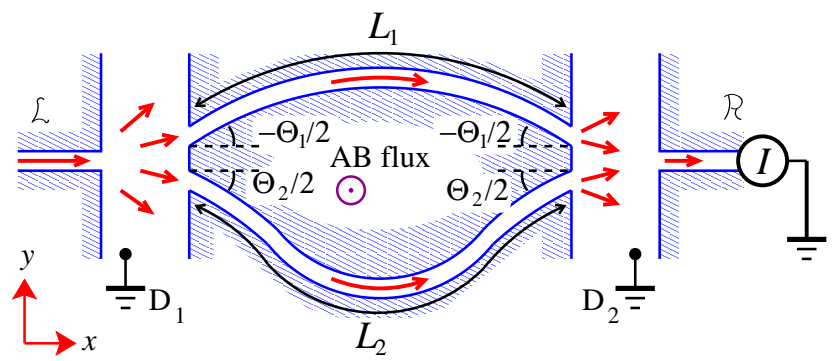

FIG. 1: The geometries we consider, with applied field, $B$ and Rashba field, $B_{\mathrm{R}}$. In (c), the BP changes sign for wires reflected in the $x$-axis $(\Theta \rightarrow-\Theta)$. In $(\mathrm{d})$ the source injects a superposition of $\uparrow$ and $\downarrow$ eigenmodes, and the spin-flip takes $\uparrow \leftrightarrow \downarrow$. In (e) spin-polarized electrons are injected at $\mathcal{L}$. Some are detected at $\mathcal{R}$, but most escape into $D_{1,2}$.

eigenmodes have momenta, $p_{\sigma}$, given by

$$
\begin{aligned}
0=p_{\sigma}^{4}- & {\left[2 p_{0}^{2}+4\left(\hbar / l_{\mathrm{R}}\right)^{2}\right] p_{\sigma}^{2}+\left[2 \hbar m B_{y} / l_{\mathrm{R}}\right] p_{\sigma} } \\
+ & p_{0}^{4}-(m B)^{2},
\end{aligned}
$$

where $B=|\mathbf{B}|$ and $p_{0}=\left[2 m\left(E_{\mathrm{F}}-E_{0}\right)\right]^{1 / 2}$. Here $\sigma= \pm 1=(\uparrow, \downarrow)$ is the spin-state, orientated along the (a) sketch of dispersion curve

(b) field felt by spin at

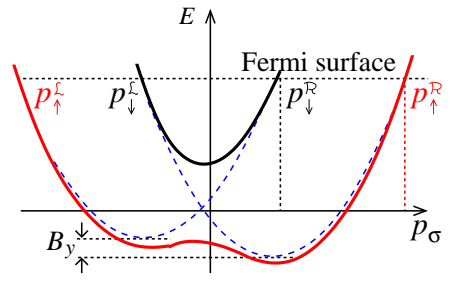
Fermi energy $\left(B_{x}=B_{y}=0\right)$

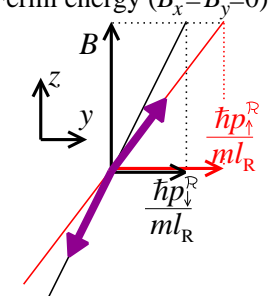

FIG. 2: (a) A sketch of the dispersion curve for $\hat{\mathcal{H}}$ in Eq. (1); dashed parabolas have $B_{x}=B_{z}=0$ [13]. (b) The effective field felt by spin-states at $E_{\mathrm{F}}$ (for $B_{x}=B_{y}=0$ ). Since $p_{\uparrow}^{\mathcal{R}}>p_{\downarrow}^{\mathcal{R}}$, the two states have non-orthogonal spins (their overall orthogonality is due to $\left.p_{\uparrow}^{\mathcal{R}} \neq p_{\downarrow}^{\mathcal{R}}\right)$.

effective field $\left(B_{x}, 2 \hbar\left(m l_{\mathrm{R}}\right)^{-1} p_{\sigma}+B_{y}, B_{z}\right)$, see Fig. 2.

Let us first consider $B_{x}=B_{z}=0$, then the solutions of Eq. (2) are $p_{\sigma}^{\mathcal{R}, \mathcal{L}}=\left(\hbar / l_{\mathrm{R}}\right) \sigma \pm\left[p_{0}^{2}+\left(\hbar / l_{\mathrm{R}}\right)^{2}+m B_{y} \sigma\right]^{1 / 2}$ with the upper and lower sign for $\mathcal{R}$ and $\mathcal{L}$ movers, respectively. We assume $p_{0}^{2}>m B_{y}$, then an $\mathcal{R}$ or $\mathcal{L}$ mover in a superposition of two spin-states, $\uparrow$ and $\downarrow$ (e.g. spin in the $x$-direction), acquires a phase difference of $\Phi^{\mathcal{R}, \mathcal{L}}=\left(\left|p_{\uparrow}^{\mathcal{R}, \mathcal{L}}\right|-\left|p_{\downarrow}^{\mathcal{R}, \mathcal{L}}\right|\right) L / \hbar$ between the two spin-states when traversing the wire. There is no energyterm because both states have the same energy. Given $p_{\sigma}^{\mathcal{R}, \mathcal{L}}$ we have $\Phi^{\mathcal{R}, \mathcal{L}}=\left(\Lambda_{B_{y}}^{-1}-\Lambda_{-B_{y}}^{-1} \pm 2 l_{\mathrm{R}}{ }^{-1}\right) L$ where the length scale $\Lambda_{B} \equiv \hbar\left[p_{0}^{2}+\left(\hbar / l_{\mathrm{R}}\right)^{2}+m B\right]^{-1 / 2}$. Noise smears this phase difference causing dephasing. We consider Gaussian-distributed noise in $\mathbf{B}$ and $l_{\mathrm{R}}$ which is much slower than the time-of-flight between source and detector. We use $\langle\cdots\rangle$ to indicate taking the expectation value, and averaging over $\delta B_{i}, \delta l_{\mathrm{R}}$ with weight $\exp \left[-\frac{1}{2} \sum_{i}\left(\delta B_{i} / \Delta_{i}\right)^{2}-\frac{1}{2}\left(\delta l_{\mathrm{R}} / \Delta_{\mathrm{R}}\right)^{2}\right]$. Assuming that the noise is weak, $\Delta_{i} \ll B_{i}$ and $\Delta_{\mathrm{R}} \ll l_{\mathrm{R}}$, we can expand $\Phi^{\mathcal{L}, \mathcal{R}}$ to first order in $\delta B_{i}$ and $\delta l_{\mathrm{R}}$. Defining $\hat{\sigma}_{1}$ as along the eigenbasis (here the $y$-axis), we have

$$
\begin{aligned}
& \left\langle\hat{\sigma}_{2,3}\right\rangle \propto\left|\left\langle\exp \left[\mathrm{i} \Phi^{\mathcal{R}, \mathcal{L}}\right]\right\rangle\right| \\
& =\exp \left[-\frac{1}{8}\left(L\left(\Lambda_{B_{y}}+\Lambda_{-B_{y}}\right) m \Delta_{B} / \hbar^{2}\right)^{2}\right] \\
& \quad \times \exp \left[-\frac{1}{2}\left(L\left(\Lambda_{B_{y}}-\Lambda_{-B_{y}} \pm 2 l_{\mathrm{R}}\right) \Delta_{\mathrm{R}} / l_{\mathrm{R}}{ }^{3}\right)^{2}\right]
\end{aligned}
$$

This yields a Gaussian decay of the purity with $L$, i.e. with the time-of-flight along the wire [14]. Such Gaussian decays are typical of slow noise (inhomogeneous broadening) [15]. Noise in $B$ dephases the spin of $\mathcal{R}$ and $\mathcal{L}$ movers in the same way (symmetric). However noise in $l_{\mathrm{R}}$ dephases $\mathcal{R}$ movers differently from $\mathcal{L}$ movers [upper vs. lower sign in Eq. (3)]. This asymmetry can only be large if $\left|\Lambda_{B_{y}}-\Lambda_{-B_{y}}\right| \sim 2 l_{\mathrm{R}}$.

In contrast, for $B_{y}=0$, Eq. (2) is a quadratic equation for $p_{\sigma}^{2}$. For every $\mathcal{L}$ mover with momentum $p_{\sigma}$ there is a $\mathcal{R}$ mover with momentum $-p_{\sigma}$. In this case $\Phi^{\mathcal{R}}=\Phi^{\mathcal{L}}$, so there is no asymmetry in dephasing.

Geometric dephasing in a curved wire, see 


\begin{tabular}{|c|c|c|c|c|}
\hline Wire & $l_{\mathrm{R}}$-noise (cf. Eq. (10) & $B_{z}$-noise & $B_{y}$-noise & $B_{x}$-noise \\
\hline Straight, finite $B_{y}$ & sym. + weak asym. & none & sym. & none \\
\hline Straight, finite $B_{z}$ & sym. & sym. & sym. & sym. \\
\hline Straight, finite $B_{x}$ & sym. & sym. & sym. & sym. \\
\hline Curved, finite $B_{z}$ & $\begin{array}{l}\text { sym. + weak geom.-induced asym. } \\
+ \text { weak purely geometric sym. }\end{array}$ & $\begin{array}{l}\text { sym. + weak geom.-induced asym. } \\
+ \text { weak purely geometric sym. }\end{array}$ & & \\
\hline Curved + spin-echo & $\begin{array}{l}\text { sym. + strong geom.-induced asym. } \\
+ \text { strong purely geometric sym. }\end{array}$ & $\begin{array}{l}\text { sym. + strong geom.-induced asym. } \\
+ \text { strong purely geometric sym. }\end{array}$ & & \\
\hline $\begin{array}{l}\text { AB set-up, } \\
\text { finite } B_{z}\end{array}$ & $\begin{array}{l}\text { sym. + strong geom.-induced asym. } \\
+ \text { strong purely geometric sym. }\end{array}$ & $\begin{array}{c}\text { sym. + weak asym. } \\
+ \text { weak geom.-induced asym. } \\
+ \text { weak purely geometric sym. }\end{array}$ & & \\
\hline
\end{tabular}

TABLE I: Contributions to dephasing for the various systems: "none" indicates the absence of dephasing [14]; "sym./asym." indicate that the terms are symmetric/asymmetric under right movers $\leftrightarrow$ left movers. Terms labelled "strong"( "weak") are of similar size to (much less than) the main symmetric term, for typical experimental parameters. "Geom.-induced" and "purely geometric" indicate terms which vanish for a straight wire, the former go like $L \Theta$ while the latter go like $\Theta^{2}$.

Fig. 1b. The electron travelling along the wire is subject to a spatially varying effective field $\left(\mathbf{B}+\mathbf{B}_{\mathrm{R}}\right.$, where $\left.\mathbf{B}=B_{z} \mathbf{e}_{z}\right)$. We go to cylindrical coordinates $(r, \theta, z)$ [16] dropping terms that go like $\lambda_{\mathrm{F}} / r$. Transforming the spinor using $\hat{\mathcal{U}}_{\theta}=\exp \left[\mathrm{i} \frac{1}{2} \theta \hat{\sigma}_{z}\right]$ gives the Hamiltonian

$$
\hat{\mathcal{H}}_{\mathrm{cyl}}=\frac{\hat{p}_{\theta}^{2}}{2 m}+E_{0}-\frac{\hbar \hat{p}_{\theta}}{m l_{\mathrm{R}}} \hat{\sigma}_{r}-\left(B_{z}+\frac{\hbar \hat{p}_{\theta}}{2 m r}\right) \hat{\sigma}_{z}
$$

where the $\theta$-dependence of the frame causes the $\hat{p}_{\theta} \hat{\sigma}_{z}$ term. The eigenmodes' momenta, $p_{\sigma} \equiv p_{\theta}(\sigma)$, are given by Eq. (22) with $B_{y}=B_{x}=0$ and $B_{z}$ replaced by $\left(B_{z}+\right.$ $\left.(2 m r)^{-1} p_{\sigma}\right)$. We next assume a large radius of curvature, $r \gg \hbar p_{0}\left[\left(\hbar p_{0} / l_{\mathrm{R}}\right)^{2}+\left(m B_{z} / 2\right)^{2}\right]^{-1 / 2}$ (so the frame's angular velocity $\ll$ precession rate in the effective field) and expand $p_{\sigma}= \pm\left|p_{\sigma}^{\infty}\right|+\hbar c_{\sigma} r^{-1}$, where $p_{\sigma}^{\infty}$ is the momentum for $r=\infty$. To order $1 / r$, we obtain $c_{\sigma}=\sigma \cos \kappa$ where we define $\kappa$ as the angle between the $z$-axis and an effective field $\left(0,2 \hbar\left[p_{0}^{2}+\left(\hbar / l_{\mathrm{R}}\right)^{2}\right]^{1 / 2} /\left(m l_{\mathrm{R}}\right), B_{z}\right)$. This is readily generalized to other wire shapes (cf. Fig. 1c), with $r$ varying along the wire coordinate, $\tilde{x},(r(\tilde{x})$ to be kept large). Noting that $\left(c_{\uparrow}-c_{\downarrow}\right)=2 c_{\uparrow}$, we obtain

$$
\begin{aligned}
\Phi^{\mathcal{R}, \mathcal{L}} & =\hbar^{-1} \int\left(\left|p_{\uparrow}^{\mathcal{R}, \mathcal{L}}\right|-\left|p_{\uparrow}^{\mathcal{R}, \mathcal{L}}\right|\right) r(\tilde{x}) \mathrm{d} \theta(\tilde{x}) \\
& =\left(\left|p_{\uparrow}^{\infty}\right|-\left|p_{\downarrow}^{\infty}\right|\right) L / \hbar \pm 2 c_{\uparrow} \Theta
\end{aligned}
$$

The first term in $\Phi^{\mathcal{R}, \mathcal{L}}$ goes like the wire length, $L$, (i.e. proportional to the time-of-flight along the wire) and is hence a dynamic phase. The second term is proportional to the total change in angle, $\Theta$, is independent of the time-of-flight and is thus a Berry (geometric) phase; it is the same for all wires in Fig. 17. As $\Theta$ is a directed angle, $\Theta \rightarrow-\Theta$ means a curve in the opposite sense (i.e. clockwise $\rightarrow$ counter-clockwise). Introducing noise (in $B_{z}$ and $l_{\mathrm{R}}$ ) now adds the factor $\left[\delta l_{\mathrm{R}} \frac{\mathrm{d}}{\mathrm{d} l_{\mathrm{R}}}+\delta B_{z} \frac{\mathrm{d}}{\mathrm{d} B_{z}}\right]\left[\left(\left|p_{\uparrow}^{\infty}\right|-\left|p_{\downarrow}^{\infty}\right|\right) L / \hbar \pm 2 c_{\uparrow} \Theta\right]$ to $\Phi_{\mathcal{R}, \mathcal{L}}$. Averaging over this noise as before yields decay (dephas- ing) of the purity with the exponent

$$
-\frac{1}{2}\left(\left[\Delta_{\mathrm{R}} \frac{\mathrm{d}}{\mathrm{d} l_{\mathrm{R}}}+\Delta_{B_{z}} \frac{\mathrm{d}}{\mathrm{d} B_{z}}\right]\left[\left(\left|p_{\uparrow}^{\infty}\right|-\left|p_{\downarrow}^{\infty}\right|\right) L / \hbar \pm 2 c_{\uparrow} \Theta\right]\right)^{2}
$$

The $\sim L^{2}$ and $\sim \Theta^{2}$ terms in the exponential (dynamic and purely geometric terms respectively) are both unchanged under $\mathcal{R} \leftrightarrow \mathcal{L}$. The cross term, $\sim L \cdot \Theta$, (a mixed dynamic-geometric term) changes sign under $\mathcal{R} \leftrightarrow \mathcal{L}$, causing a (geometry-induced) left-right asymmetry.

Coherent oscillations carry the phase of Eq. (5), their amplitude decays with the exponent in Eq. (6). Ambiguity in choosing the measurement axis (any axis perpendicular to the axis of the eigenbasis) causes ambiguity in the phase (know as gauge-dependence), but not the amplitude. Thus the geometric contribution to dephasing is gauge-independent even when the $\mathrm{BP}$ is not [6].

Spin-echo. To maximize the geometric or asymmetric effects one may use a spin-echo technique, sketched in Fig. 18. If $\Theta=\Delta L=0$, the wires left and right of the spin-flipper are identical, and any spin-component acquires opposite phases before and after the spin-flip. Thus for non-zero $\Theta$ and $\Delta L$, the phase is given by Eq. (5) with $L$ replaced by $\Delta L$. Dephasing is given by Eq. (6) with $L \rightarrow \Delta L$. Varying $\Delta L$ changes the relative size of the $\Delta L^{2}, \Delta L \Theta$ and $\Theta^{2}$ contributions to dephasing. For $\Delta L=0$, there is only purely geometric dephasing. Asymmetry is maximized for a small $\Delta L$, such that the $\Delta L^{2}$ and $\Delta L \Theta$ terms are similar in size.

Aharonov-Bohm interferometer. Measuring the flux-sensitive current through such an interferometer (Fig. 11) allows us to study dephasing [17], while avoiding the need to measure spin-components of the current. We generate right or left movers via a voltage bias to either the $\mathcal{L}$ or the $\mathcal{R}$ lead. Asymmetry in dephasing will manifest itself as a difference in the visibility (magnitude of the $\mathrm{AB}$ oscillations in the current) between right and left movers, We assume that the multi-terminal (open) interferometer is sufficiently open that no higher windings 
around it occur. This also avoids the symmetry constraints imposed by the two-terminal Onsager-Büttiker relations. We consider injected electrons which are spinpolarized along $\mathbf{B}=B_{z} \mathbf{e}_{z}$, thus the phase difference between the two paths is $\Phi^{\mathcal{R}, \mathcal{L}}= \pm \Phi_{\mathrm{AB}}+\left|p_{\uparrow}^{\infty}\right| L / \hbar \pm c_{\uparrow} \Theta$ where $L=L_{2}-L_{1}$ and $\Theta=\Theta_{2}-\Theta_{1}$. The AharonovBohm phase $\Phi_{\mathrm{AB}}=-e B_{z} \mathcal{A} /\left(g \mu_{\mathrm{B}} \hbar\right)$ where $\mathcal{A}$ is the area enclosed by the paths. The current at the detector is

$$
\left|I^{\mathcal{R}, \mathcal{L}}\right|=\left|I_{1}\right|+\left|I_{2}\right|+2\left|I_{1} I_{2}\right|^{1 / 2} A^{\mathcal{R}, \mathcal{L}} \cos \Phi^{\mathcal{R}, \mathcal{L}}
$$

where $I_{i}$ is the part of the current in arm $i$ which enters the detector lead. In the absence of noise $A^{\mathcal{R}, \mathcal{L}}=1$. For $I_{1}=I_{2}$, the visibility of the AB oscillations is maximal. Averaging over noise in $B_{z}$ and $l_{\mathrm{R}}$, we find that

$$
\begin{aligned}
A^{\mathcal{R}, \mathcal{L}}=\exp & {\left[-\frac{1}{2} \Delta_{B_{z}}^{2}\left(\frac{e \mathcal{A}}{g \mu_{\mathrm{B}} \hbar} \pm \frac{L}{\hbar} \frac{\mathrm{d}\left|p_{\uparrow}^{\infty}\right|}{\mathrm{d} B_{z}}+\Theta \frac{\mathrm{d} c_{\uparrow}}{\mathrm{d} B_{z}}\right)^{2}\right.} \\
& \left.-\frac{1}{2} \Delta_{\mathrm{R}}^{2}\left( \pm \frac{L}{\hbar} \frac{\mathrm{d}\left|p_{\uparrow}^{\infty}\right|}{\mathrm{d} l_{\mathrm{R}}}+\Theta \frac{\mathrm{d} c_{\uparrow}}{\mathrm{d} l_{\mathrm{R}}}\right)^{2}\right]
\end{aligned}
$$

This is asymmetric, with the upper (lower) sign for $\mathcal{R}(\mathcal{L})$ movers. There are contributions to dephasing due to the curvature, $\sim L \Theta$ (asymmetric) and $\sim \Theta^{2}$ (symmetric).

Discussion. Quantum wires in $\mathrm{Ga}_{x} \mathrm{In}_{1-x} \mathrm{As} / \mathrm{InP}$ have $l_{\mathrm{R}} \simeq 5 \lambda_{\mathrm{F}} \simeq 200 \mathrm{~nm}[18$. Then the ratio of asymmetric to total dephasing in a straight-wire (cf. Eq. (3)) will be small, equal to $2 \lambda_{\mathrm{F}}^{2} /\left(l_{\mathrm{R}} l_{B}\right) \sim 8 \%$, where $l_{B}=v_{\mathrm{F}} \hbar / B \sim$ $l_{\mathrm{R}}$. However for spin-echo set-ups with $\Delta L \sim l_{\mathrm{R}}$, this ratio can be of order one (cf. Eq. (6) and thereafter). Then the dephasing is not very strong, nonetheless we estimate that one can tune $\Delta L$ such that $\mathcal{R}$ movers lose at least $50 \%$ of their coherence (purity $P<3 / 4$ ), while $\mathcal{L}$ movers are not dephased. For the AB set-up with $l_{\mathrm{R}^{-}}$ noise (cf. Eq. (8)), the situation is the same as for the spin-echo (with $L$ playing the role of $\Delta L$ ), while for $B_{z^{-}}$ noise the ratio of asymmetric to total dephasing (in the exponent) is tiny $\sim \lambda_{\mathrm{F}} L / \mathcal{A}$.

To summarize, we have analyzed the effect of noise on current-carrying electrons, both spinful and spinpolarized, subject to a spin-orbit interaction. The dephasing may be measured through the spin-components of the emerging electrons, or the visibility of AB oscillations. We have demonstrated both geometric dephasing and left-right asymmetry in dephasing. The noise studied here fluctuates at a pace slower than the timeof-flight, leading to dephasing with the exponential of $\sim L^{2}, \sim L \cdot \Theta, \sim \Theta^{2}$. By contrast, for faster fluctuations (studied for a spin or qubit in a time-dependent field [6]), the dephasing would be exponential with $L, \Theta$, see also [19, 20, 21]. Other $\mathcal{L} \leftrightarrow \mathcal{R}$ asymmetries occur in real devices, however those discussed here have a unique signature; they are controlled by the noise-power.

We thank Yu. Makhlin and G. Schön for very useful discussions. This work was supported by Swiss
NSF, FG Schwerpunktprogramm 1285 "Halbleiter Spintronik", EU Transnational Access program (RITA-CT2003-5060965), US-Israel BSF, Minerva Foundation and Albert Einstein Minerva Center for Theoretical Physics.

[1] Semiconductor Spintronics and Quantum Computation, edited by D.D. Awschalom, D. Loss, and N. Samarth (Springer, Berlin, 2002).

[2] E.I. Rashba, in "Future Trends in Microelectronics. Up to Nano Creek", Ed. by S. Luryi, J. M. Xu, and A. Zaslavsky (Wiley, Hoboken, 2007), cond-mat/0611194

[3] H.-A. Engel, L.P. Kouwenhoven, D. Loss and C.M. Marcus, Quantum Information Processing 3, 115 (2004).

[4] A. Cottet et al, Semicond. Sci. Technol. 21, S78 (2006).

[5] Y. Meir, Y. Gefen and O. Entin-Wohlman, Phys. Rev. Lett. 63, 798 (1989). D. Loss, P. Goldbart and A.V. Balatsky, Phys. Rev. Lett. 65, 1655 (1990). A.G. Aronov and Y.B. Lyanda-Geller Phys. Rev. Lett. 70, 343 (1993).

[6] R.S. Whitney, Y. Makhlin, A. Shnirman and Y. Gefen, Phys. Rev. Lett. 94, 070407 (2005).

[7] The gate RC times $\gtrsim 10^{-6} \mathrm{~s}$ (although they may be engineered to be shorter), while the time-of-flight along ballistic wires $\left(L<0.1 \mathrm{~mm}, v_{\mathrm{F}} \sim 10^{5} \mathrm{~m} / \mathrm{s}\right)$ is $\lesssim 10^{-9} \mathrm{~s}$.

[8] R. Lassnig, Phys. Rev. B, 31, 8076 (1985). W. Zawadzki and P. Pfeffer, Semicond. Sci. Technol. 19, R1 (2004). E. Bernardes, J. Schliemann, M. Lee, J. Carlos Egues, and D. Loss, Phys. Rev. Lett. 99, 076603 (2007).

[9] J. Nitta, T. Akazaki, H. Takayanagi and T. Enoki, Phys. Rev. Lett. 78, 1335 (1997). G. Engels, J. Lange, Th. Schäpers, and H. Lüth, Phys. Rev. B 55, R1958 (1997).

[10] This equation for $P$ is approximate when Fermi-surface states have slightly non-orthogonal spins, Fig 2 b.

[11] Equal Rashba and Dresselhaus terms do not suppress dephasing in our model, cf. J. Schliemann, J. Carlos Egues and D. Loss, Phys. Rev. Lett. 90, 146801 (2003).

[12] This term reads $\mathcal{H}_{y}=(2 m)^{-1} \hat{p}_{y}^{2}+\left(m l_{\mathrm{R}}\right)^{-1} \hat{p}_{y} \hat{\sigma}_{x}+V(y)$. For $\lambda_{\mathrm{F}} \ll l_{\mathrm{R}}$, this is a fast oscillator coupling to the slow spin's $x$-component. It slightly reduces the effective fields in $y, z$-directions, we absorb this into $\mathbf{B}, \mathbf{B}_{\mathrm{R}}$.

[13] M. Governale and U. Zülicke, Phys. Rev. B 66, 073311 (2002).

[14] Weak-noise in $x$ or $z$, with $B_{x}=B_{z}=0$ on average, gives only $\left(2^{\text {nd }}\right.$ order $)$ dephasing, $\mathcal{O}[\hbar / p L]$ smaller.

[15] see e.g. Spin dynamics: Basics of nuclear magnetic resonance, M.H. Levitt (Wiley, New York, 2001). A. Kamenev and Y. Gefen, Phys. Rev. B 54, 5428 (1996).

[16] F.E. Meijer, A.F. Morpurgo and T.M. Klapwijk, Phys. Rev. B 66, 033107 (2002)

[17] F. Marquardt and C. Bruder, Phys. Rev. B 65, 125315 (2002)

[18] Th. Schäpers, V.A. Guzenko, M.G. Pala, U. Zülicke, M. Governale, J. Knobbe, and H. Hardtdegen, Phys. Rev. B 74, 081301(R) (2006).

[19] P. San-Jose, G. Zarand, A. Shnirman, G. Schön, Phys. Rev. Lett. 97, 076803 (2006).

[20] R.S. Whitney and Y. Gefen, Phys. Rev. Lett. 90, 190402 (2003). R.S. Whitney, Yu. Makhlin, A. Shnirman and Y. Gefen, cond-mat/0401376.

[21] P.J. Leek et al, Science 318, 1889 (2007). 